
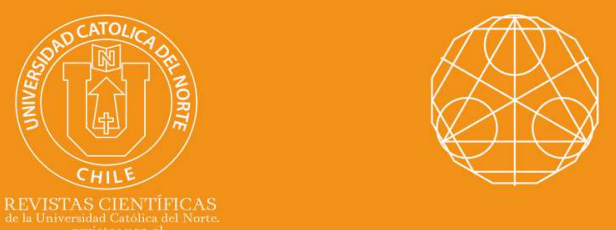

\title{
Multiplicative degree based topological indices of some chemical structures in drug
}

\author{
Murat Cancan ${ }^{1}$ (1) orcid.org/0000-0002-8606-2274 \\ Sourav Mondal ${ }^{2}$ (D) orcid.org/0000-0003-1928-7075 \\ Nilanjan De ${ }^{3}$ (10) orcid.org/0000-0001-9143-7045 \\ Anita Pal ${ }^{4}$ (1) orcid.org/0000-0002-2514-5463
}

${ }^{1}$ Van Yüzüncü Yıl University, Faculty of Education, Van, Turkey.

mancan@yyu.edu.tr

National Institute of Technology Durgapur, Dept. of mathematics, Durgapur, WB, India.

2】 souravmath94@gmail.com; 4 anita.buie@gmail.com

${ }^{3}$ Calcutta Institute of Engineering \& Management, Dept of BasicSci and Humanities, WB, Kolkata, India.

ve.nilanjan@rediffmail.com

\section{Received: April 2020 | Accepted: August 2020}

\section{Abstract:}

In quantitative structure property relationship analysis (QSPR) and quantitative structure property relationship analysis (QSAR) the correlation between different properties/activities and molecular structure of chemical compounds is investigated which is helpful in drug design. Topological index is an useful tool to predict different physical and chemical properties of molecule by collecting information from the molecular graph. In this article, multiplicative degree based topological indices are obtained for some chemical structures widely used in drug design, especially in anticancer drug discovery. To visualize the indices, the results are interpreted graphically.

Keywords: Topological index; Dox-loaded micelle comprising PEGPAsp block copolymer with chemically conjugated Dox $\mathrm{SP}[\mathrm{n}]$; Polyomino chain of $\mathrm{n}$-cycles; Triangular benzenoid $\left(\mathrm{T}_{n}\right)$; Circumcoronene benzenoid series $\left(\mathrm{H}_{\mathrm{k}}\right)$.

MSC (2020): 05C35, 05C07, 05C40.

\section{Cite this article as (IEEE citation style):}

M. Cancan, S. Mondal, N. De, and A. Pal, "Multiplicative degree based topological indices of some chemical structures in drug", Proyecciones (Antofagasta, On line), vol. 39, no. 5, pp. 1347-1364, Oct. 2020, doi: 10.22199/issn.0717-6279-2020-05-0082.

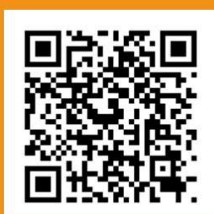

Article copyright: (c) 2020 Murat Cancan, Sourav Mondal, Nilanjan De, and Anita Pal. This is an open access article distributed under the terms of the Creative Commons Licence, which permits unrestricted use and distribution provided the original author and source are credited. 


\section{Introduction}

With rapid development in chemical field, it is very difficult to perform chemical experiments which increase cost and time to determine properties of particular compound. So, a new technique called chemical graph theory evolved. The chemical graph theory is one of the most popular subjects in the field of graph theory. In chemical graph theory, we deal with molecular graph [1], a simple connected graph, to determine physiochemical and psychochemical properties of particular compound. In this field of research, topological index plays a prominent role to study the topological properties of different chemical structures. Topological index is a numerical parameter associated with the molecular graph of chemical compound. It remains unchanged under graph isomorphism. Now a days lot of research work is presented in chemical graph theory particularly in investigating topological properties of chemically significant composite graphs. Gao et al. [2] have derived degree based topological indices for the family of smart polymers which is widely used in anticancer drugs manufacturing. Kwun et al. [3] obtained multiplicative degree based topological indices of some silicon-carbon structures. Topological properties of some chemical structures used in drug design have been studied in $[4,5]$. For more works on this field, readers are referred to $[6,7,8,9,10,11,12]$. In this paper we have computed some multiplicative degree based topological indices of chemical compounds widely used in drug discovery, especially, the family of smart polymers to produce anticancer drugs which is a modern topic of research in medical science. Further, we have considered polyomino chain of n-cycle, triangular benzenoid $\left(T_{n}\right)$ and circumcoronene benzenoid series $\left(H_{k}\right)$.

\section{Some basic definitions}

Throughout this paper, we consider simple connected graph. Let $V(G)$ and $E(G)$ be the vertex and edge sets of a graph $G$, respectively. The degree of a vertex $v \in V(G)$, denoted by $d(v)$, is the count of edges incident to $v$. The cycle graph with $n$ nodes is denoted by $C_{n}$. Wang et al. defined a general multiplicative index [13] as follows:

$$
W_{1}^{s}(G)=\prod_{u \in V(G)}(d(u))^{s} .
$$

Where $s$ is real number. For $s=1,2$, in (2.1), the Narumi-Katayama 
index [14] and the first multiplicative Zagreb index [15] are defined respectively, as follows:

$$
N K(G)=\prod_{u \in V(G)} d(u), \quad \Pi_{1}(G)=\prod_{u \in V(G)}(d(u))^{2} .
$$

Kulli et al. introduced two more indices known as first and second generalized multiplicative Zagreb indices [16] which are defined as follows:

$$
\begin{gathered}
M Z_{1}^{a}(G)=\prod_{u v \in E(G)}(d(u)+d(v))^{a}, \\
M Z_{2}^{a}(G)=\prod_{u v \in E(G)}(d(u) d(v))^{a} .
\end{gathered}
$$

Where $a$ is real number. Considering $a=1,2,-1 / 2$ in $(2.2)$, new version of the multiplicative first Zagreb index [17], first multiplicative hyperZagreb index [18], multiplicative sum connectivity index [19] are defined respectively, as follows:

$$
\begin{gathered}
\Pi_{1}^{*}(G)=\prod_{u v \in E(G)}(d(u)+d(v)), \quad H \Pi_{1}(G)=\prod_{u v \in E(G)}(d(u)+d(v))^{2}, \\
S C I \Pi(G)=\prod_{u v \in E(G)}(d(u)+d(v))^{-\frac{1}{2}}
\end{gathered}
$$

Putting $a=1,2,-\frac{1}{2}$ in (2.3), second multiplicative Zagreb index [15], second multiplicative hyper-Zagreb index [18], and multiplicative product connectivity index [19] are defined respectively as follows:

$$
\begin{gathered}
\Pi_{2}(G)=\prod_{u v \in E(G)} d(u) d(v), \quad H \Pi_{2}(G)=\prod_{u v \in E(G)}(d(u) d(v))^{2}, \\
P C \Pi(G)=\prod_{u v \in E(G)}(d(u) d(v))^{-\frac{1}{2}} .
\end{gathered}
$$

Multiplicative atomic bond connectivity index and multiplicative Geometric arithmetic index are defined [3] respectively as follows:

$$
A B C(G)=\prod_{u v \in E(G)} \sqrt{\frac{d(u)+d(v)-2}{d(u) d(v)}}, \quad G A H(G)=\prod_{u v \in E(G)} \frac{2 \sqrt{d(u) d(v)}}{d(u)+d(v)} .
$$




\section{Motivation}

Smart polymers are composed of high performance polymers that change its physiochemical properties according to the environment they are in. Thus smart polymers are stimuli-responsive polymers. Polymeric micelles are most accepted modalities of macro-molecular drug delivery systems. It gives unique effect on body for drug targeting, tumor accumulation. Polymeric micelle has advantages such as controlled drug release, high drug loading capacity, unnecessariness of chemical structure modification of the drug etc [20]. PEG is widely used hydrophilic polymer for drug delivery. The hydrated PEG shell increase stability and enhance blood flow times of drug that is protected by or bound to PEG. The Polymer, PEG-bPASP-DOX conjugation has been accomplished by means of acid cleavable hydrazones linkages [21]. Over the past few years, polymeric micelles of DOX conjugated poly (ethylene glycol)-b-poly (aspirate) (PEG-b-PASP) block copolymers have gained considerable attention because of their ease in the formation of micelles in water [22]. The Dox-loaded micelle containing PEG-PAsp block copolymer with chemically conjugated Dox $(S P[n])$, depicted in Figure 3.1, is a famous family of smart polymer which is used as anthracycline anticancer antibiotic and applied in treating various kinds of cancers.

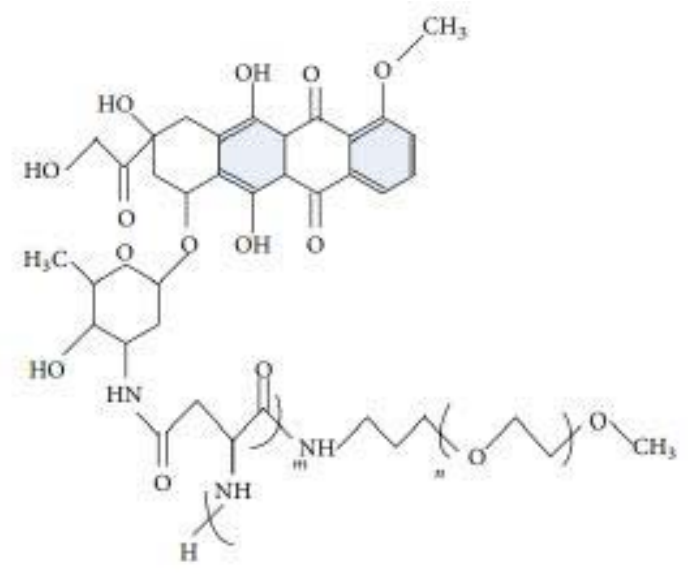

Figure 3.1: Chemical structure of $S P[n]$. 


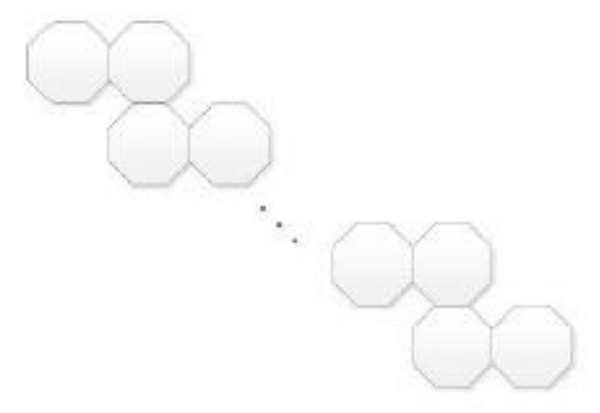

Figure 3.2: The zig-zag chain of 8-cycles.

A polyomino system is a finite 2-connected planar graph so that a regular $C_{4}$ cycle of length one surrounds the internal cell. In polyomino system two nodes are adjacent iff two cells share a common edge [23]. Its structure is depicted in Figure 3.2. Polyomino systems have several applications in crystal physics [24]. We also consider graph of triangular benzenoid $T_{n}$ in which $n$ is number of hexagonal structure in base graph. We clearly observe from Figure 3.3 that $T_{n}$ has $\frac{1}{2} n(n+1)$ hexagons [25]. Circumcoronene benzenoid series consist of several copies of benzene $C_{6}$ on the perimeter (Figure 3.5,3.4). After generalization from benzene molecules we get the circumcoronene series of benzenoid. Benzene is important from chemistry point of view as it helps in formation of aromatic compound.

\section{Main Results}

In this section, we focus on computing the multiplicative degree based topological indices of the molecular graphs discussed in the previous section.

4.1. Multiplicative topological indices of Dox-loaded micelle comprising PEG-PAsp block copolymer with chemically conjugated Dox $(S P[n])$ 


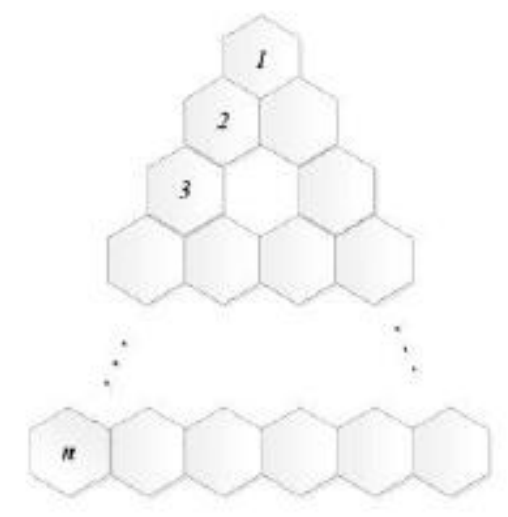

Figure 3.3: Molecular graph of triangular benzenoid.

Theorem 1. Let $G$ be the Dox-loaded micelle comprising PEG-PAsp block copolymer with chemically conjugated Dox $(S P[n])$. Then we have

(i) $W_{1}^{s}(G)=2^{s(18 n+4)} \cdot 3^{(20 n s)}$,

(ii) $M Z_{1}^{a}(G)=2^{2 a(23 n+5)} \cdot 3^{a(20 n+1)} \cdot 5^{a(19 n-1)} \cdot 7^{(a n)}$,

(iii) $M Z_{2}^{a}(G)=2^{8 a(5 n+1)} \cdot 3^{(60 a n)}$,

(iv) $A B C(G)=\left(\frac{1}{\sqrt{2}}\right)^{(27 n+4)} \cdot\left(\sqrt{\frac{2}{3}}\right)^{(9 n+1)} \cdot\left(\frac{\sqrt{3}}{2}\right)^{(n)} \cdot\left(\frac{4}{5}\right)^{(n)} \cdot\left(\frac{2}{3}\right)^{(16 n)} \cdot\left(\frac{\sqrt{5}}{2 \sqrt{3}}\right)^{(n)}$,

(v) $G A H(G)=\left(\frac{2 \sqrt{2}}{3}\right)^{(4 n+1)} \cdot\left(\frac{\sqrt{3}}{2}\right)^{(9 n+1)} \cdot\left(\frac{4}{5}\right)^{(n)} \cdot\left(\frac{2 \sqrt{6}}{5}\right)^{(18 n-1)} \cdot\left(\frac{4 \sqrt{3}}{7}\right)^{(n)}$.

Proof 1. To construct the vertex and edge partitions, we consider the following notations.

$$
\begin{gathered}
V_{i}=\{u \in V(G): d(u)=i\}, E_{i}=\{u v \in E(G): d(u)+d(v)=i\}, \\
E_{i}^{*}=\{u v \in E(G): d(u) d(v)=i\} .
\end{gathered}
$$

It is clear from the structure analysis of $G$ (Figure 3.1) that $|V(G)|=$ $49 n+6,|E(G)|=54 n+5$. The vertex and edge partitions of $G$ are shown in Table 4.1, and Table 4.2 respectively. 


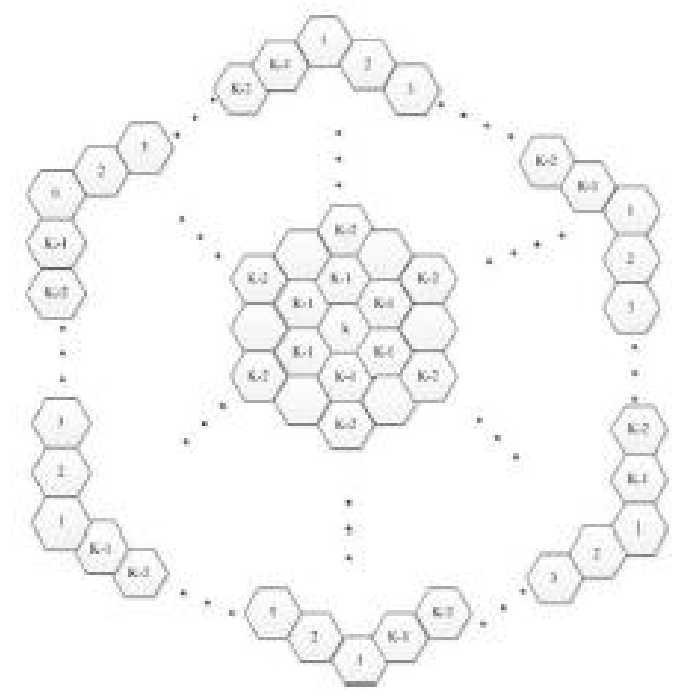

Figure 3.4: The molecular graph of $H_{k}$ for $k \geq 1$.

Therefore, using the vertex and edge partitions of $S P[n]$ in the formulation of multiplicative topological indices, we derive the following results.

$$
\begin{aligned}
W_{1}^{s}(G) & =\prod_{u \in V(G)}(d(u))^{s} \\
& =\prod_{u \in V_{1}}(d(u))^{s} \cdot \prod_{u \in V_{2}}(d(u))^{s} \cdot \prod_{u \in V_{3}}(d(u))^{s} \cdot \prod_{u \in V_{4}}(d(u))^{s} \\
& =2^{s(18 n+4)} \cdot 3^{(20 n s)}
\end{aligned}
$$



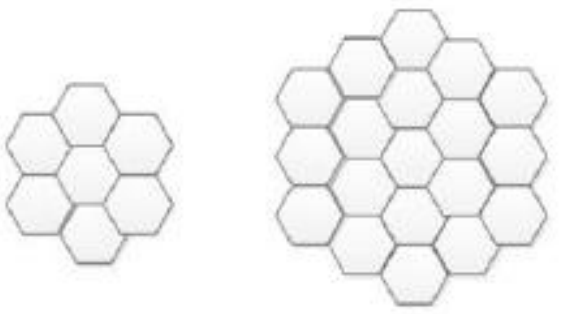

Figure 3.5: The molecular graph of $H_{1}, H_{2}$ and $H_{3}$, respectively.

Table 4.1: Vertex partition of $S P[n]$.

\begin{tabular}{ccccc}
\hline Partition of $V(G)$ & $V_{1}$ & $V_{2}$ & $V_{3}$ & $V_{4}$ \\
\hline Frequency & $2(6 n+1)$ & $4(4 n+1)$ & $20 n$ & $n$ \\
\hline
\end{tabular}

$$
\begin{aligned}
M Z_{1}^{a}(G)= & \prod_{u v \in E(G)}(d(u)+d(v))^{a} \\
= & \prod_{u v \in E_{2}^{*}}(d(u)+d(v))^{a} \cdot \prod_{u v \in E_{3}^{*}}(d(u)+d(v))^{a} \cdot \prod_{u v \in E_{4} \cap E_{4}^{*}}(d(u)+d(v))^{a} \\
& \cdot \prod_{u v \in E_{5} \cap E_{4}^{*}}(d(u)+d(v))^{a} \cdot \prod_{u v \in E_{5} \cap E_{6}^{*}}(d(u)+d(v))^{a} \cdot \prod_{u v \in E_{6} \cap E_{8}^{*}}(d(u)+ \\
& d(v))^{a} \cdot \prod_{u v \in E_{6} \cap E_{9}^{*}}(d(u)+d(v))^{a} \cdot \prod_{u v \in E_{7} \cap E_{12}^{*}}(d(u)+d(v))^{a} \\
= & {\left[3^{a}\right]^{(2 n+1)} \cdot\left[4^{a}\right]^{(9 n+1)} \cdot\left[5^{a}\right]^{(n)} \cdot\left[4^{a}\right]^{(5 n+4)} \cdot\left[5^{a}\right]^{(18 n-1)} \cdot\left[6^{a}\right]^{(2 n)} \cdot\left[6^{a}\right]^{(16 n)} } \\
& \cdot\left[7^{a}\right]^{(n)} \\
= & 2^{2 a(23 n+5)} \cdot 3^{a(20 n+1)} \cdot 5^{a(19 n-1)} \cdot 7^{(a n)} .
\end{aligned}
$$


Table 4.2: Edge partition of $S P[n]$.

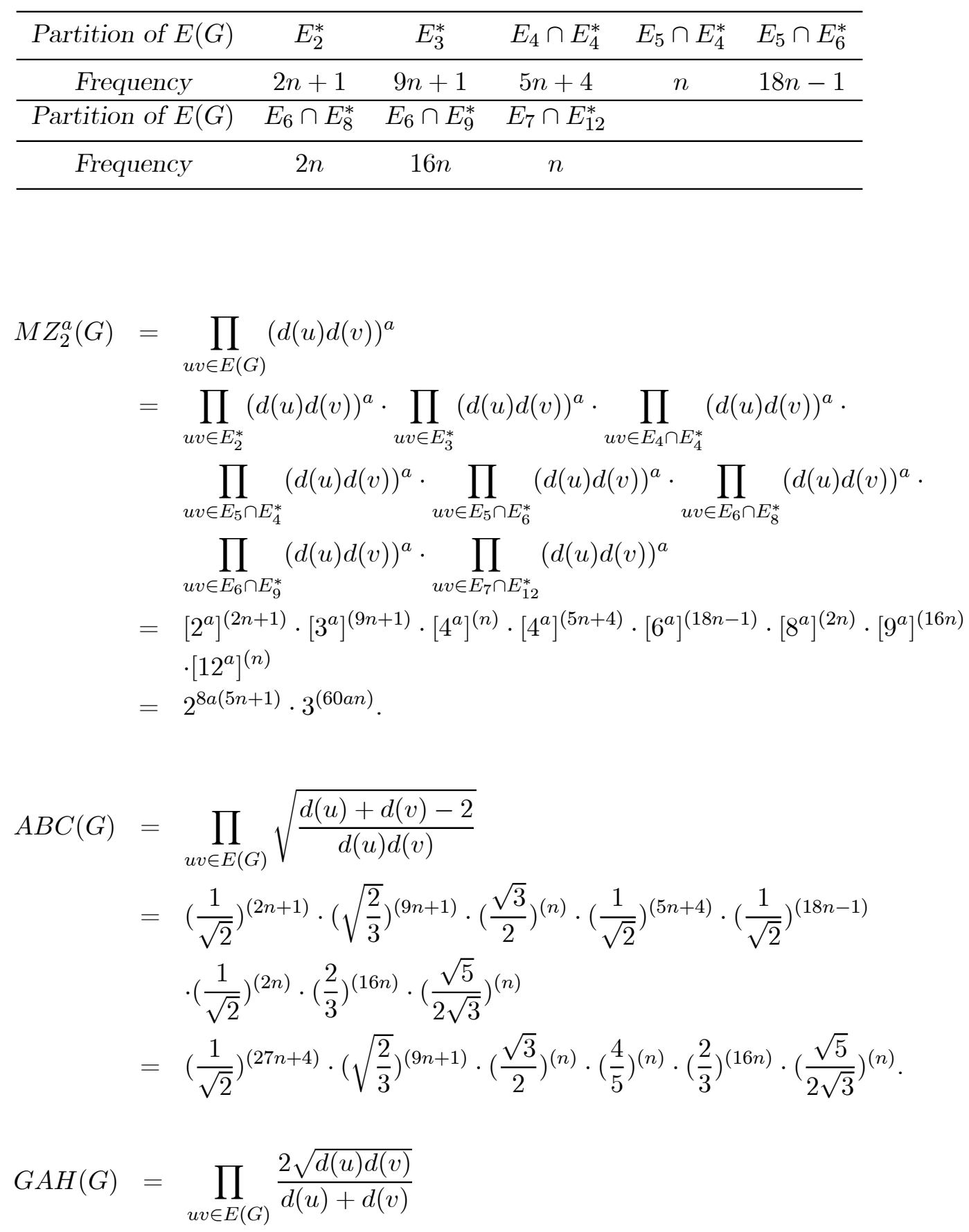




$$
\begin{aligned}
= & \left(\frac{2 \sqrt{2}}{3}\right)^{(2 n+1)} \cdot\left(\frac{\sqrt{3}}{2}\right)^{(9 n+1)} \cdot\left(\frac{4}{5}\right)^{(n)} \cdot(1)^{(5 n+4)} \cdot\left(\frac{2 \sqrt{6}}{5}\right)^{(18 n-1)} . \\
& \left(\frac{2 \sqrt{2}}{3}\right)^{(2 n)} \cdot(1)^{(16 n)} \cdot\left(\frac{4 \sqrt{3}}{7}\right)^{(n)} \\
= & \left(\frac{2 \sqrt{2}}{3}\right)^{(4 n+1)} \cdot\left(\frac{\sqrt{3}}{2}\right)^{(9 n+1)} \cdot\left(\frac{4}{5}\right)^{(n)} \cdot\left(\frac{2 \sqrt{6}}{5}\right)^{(18 n-1)} \cdot\left(\frac{4 \sqrt{3}}{7}\right)^{(n)} .
\end{aligned}
$$

Hence the proof.

Putting $s=1,2, a=1,2,-\frac{1}{2}$, in Theorem 1 , we obtain the following corollary.

Corollary 1. Different multiplicative degree based indices of $S P[n]$ are given by,

(i) $N K(G)=2^{2(9 n+2)} \cdot 3^{(20 n)}$,

(ii) $\Pi_{1}(G)=2^{4(9 n+2)} \cdot 3^{(40 n)}$,

(iii) $\Pi_{1}^{*}(G)=2^{2(23 n+5)} \cdot 3^{(20 n+1)} \cdot 5^{(19 n-1)} \cdot 7^{(n)}$,

(iv) $H \Pi_{1}(G)=2^{4(23 n+5)} \cdot 3^{2(20 n+1)} \cdot 5^{2(19 n-1)} \cdot 7^{(2 n)}$,

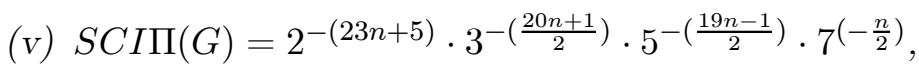

(vi) $\Pi_{2}(G)=2^{8(5 n+1)} \cdot 3^{(60 n)}$,

(vii) $H \Pi_{2}(G)=2^{16(5 n+1)} \cdot 3^{(120 n)}$,

(viii) $P C \Pi(G)=2^{-4(5 n+1)} \cdot 3^{(-30 n)}$.

\subsection{Multiplicative topological indices of polynomial chain of $n$ - cycles.}

The structure of the polynomial chain of $n$-cycles for $n=8$ is shown in the Figure 3.2. We construct the vertex and edge partitions of polynomial chain of $n$-cycles in the following tableau (Table 4.3 and Table 4.4) using the same notations as prescribed in the Theorem 1.

Now, using the vertex (Table 4.3) and edge partitions (Table 4.4) for the polynomial chain of n-cycles in the formulation of multiplicative topological indices, we derive the following results like previous.

Theorem 2. Let $G$ be the polynomial chain of $n$-cycles. Then we have 
Table 4.3: Vertex partition of the polynomial chain of $n$-cycles.

\begin{tabular}{ccc}
\hline Partition of $V(G)$ & $V_{2}$ & $V_{3}$ \\
\hline Frequency & $4(4 n+1)$ & $2(4 n-1)$ \\
\hline
\end{tabular}

Table 4.4: Edge partition of the polynomial chain of $n$-cycles.

\begin{tabular}{cccc}
\hline Partition of $E(G)$ & $E_{4} \cap E_{4}^{*}$ & $E_{5} \cap E_{6}^{*}$ & $E_{6} \cap E_{9}^{*}$ \\
\hline Frequency & $4(3 n+1)$ & $8 n$ & $2(4 n-1)$ \\
\hline
\end{tabular}

(i) $W_{1}^{s}(G)=2^{s(16 n+4)} \cdot 3^{s(8 n-2)}$,

(ii) $M Z_{1}^{a}(G)=2^{a(32 n+5)} \cdot 3^{a(8 n-3)} \cdot 5^{18 a n}$,

(iii) $M Z_{2}^{a}(G)=2^{2 a(21 n+4)} \cdot 3^{2 a(17 n-3)}$,

(iv) $A B C(G)=\left(\frac{1}{\sqrt{2}}\right)^{4(5 n+1)} \cdot\left(\frac{2}{3}\right)^{(8 n-3)}$,

(v) $G A H(G)=\left(\frac{2 \sqrt{6}}{5}\right)^{(8 n)}$.

Now putting $s=1,2, a=1,2,-\frac{1}{2}$, in Theorem 2 , we obtain the following corollary.

Corollary 2. Different multiplicative degree based indices for the polynomial chain of $n$-cycles are given by,

(i) $N K(G)=2^{4(4 n+1)} \cdot 3^{2(4 n-1)}$,

(ii) $\Pi_{1}(G)=2^{8(4 n+1)} \cdot 3^{4(4 n-1)}$,

(iii) $\Pi_{1}^{*}(G)=2^{(32 n+5)} \cdot 3^{(8 n-3)} \cdot 5^{(18 n)}$,

(iv) $H \Pi_{1}(G)=2^{2(32 n+5)} \cdot 3^{2(8 n-3)} \cdot 5^{(36 n)}$,

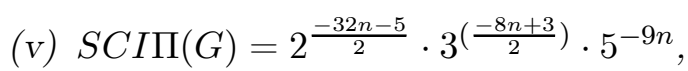

(vi) $\Pi_{2}(G)=2^{2(21 n+4)} \cdot 3^{2(17 n-3)}$,

(vii) $H \Pi_{2}(G)=2^{4(21 n+4)} \cdot 3^{4(17 n-3)}$,

(viii) $P C \Pi(G)=2^{-(21 n+4)} \cdot 3^{-(17 n-3)}$. 


\subsection{Multiplicative topological indices of the triangular benzenoid $T_{n}$.}

The vertex and edge partitions of the triangular benzenoid $T_{n}$ are shown in Table 4.5 and Table 4.6, respectively.

Table 4.5: Vertex partition of the triangular benzenoid $T_{n}$.

\begin{tabular}{ccc}
\hline Partition of $V(G)$ & $V_{2}$ & $V_{3}$ \\
\hline Frequency & $3(n+1)$ & $n^{2}+n-2$ \\
\hline
\end{tabular}

Table 4.6: Edge partition of the triangular benzenoid $T_{n}$.

\begin{tabular}{cccc}
\hline Partition of $E(G)$ & $E_{4} \cap E_{4}^{*}$ & $E_{5} \cap E_{6}^{*}$ & $E_{6} \cap E_{9}^{*}$ \\
\hline Frequency & 6 & $\frac{3 n(n-1)}{2}$ & $6(n-1)$ \\
\hline
\end{tabular}

Therefore, using the vertex and edge partitions for the triangular benzenoid $T_{n}$ in the formulation of multiplicative topological indices, we derive the following results.

Theorem 3. Let $G$ be the triangular benzenoid $T_{n}$. Then we have

(i) $W_{1}^{s}(G)=2^{s(3 n+3)} \cdot 3^{s\left(n^{2}+n-2\right)}$,

(ii) $M Z_{1}^{a}(G)=2^{6 a(n+1)} \cdot 3^{6 a(n-1)} \cdot 5^{\frac{3 a n(n-1)}{2}}$,

(iii) $M Z_{2}^{a}(G)=2^{\frac{3 a\left(n^{2}-n+8\right)}{2}} \cdot 3^{\frac{3 a(n+8)(n-1)}{2}}$,

(iv) $A B C(G)=\left(\frac{1}{\sqrt{2}}\right)^{3\left(n^{2}-n+4\right)} \cdot\left(\frac{2}{3}\right)^{6(n-1)}$,

(v) $G A H(G)=\left(\frac{2 \sqrt{6}}{5}\right)^{\frac{3 n(n-1)}{2}}$.

Now putting $s=1,2, a=1,2,-\frac{1}{2}$, in Theorem 3 , we obtain the following corollary.

Corollary 3. Different multiplicative degree based indices for the polynomial chain of $n$-cycles are given by, 
(i) $N K(G)=2^{3(n+1)} \cdot 3^{\left(n^{2}+n-2\right)}$,

(ii) $\Pi_{1}(G)=2^{6(n+1)} \cdot 3^{2\left(n^{2}+n-2\right)}$,

(iii) $\Pi_{1}^{*}(G)=2^{6(n+1)} \cdot 3^{6(n-1)} \cdot 5^{\frac{3 n(n-1)}{2}}$,

(iv) $H \Pi_{1}(G)=2^{12(n+1)} \cdot 3^{12(n-1)} \cdot 5^{3 n(n-1)}$,

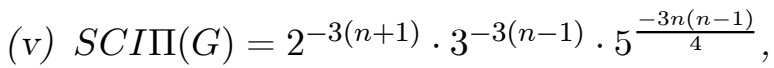

(vi) $\Pi_{2}(G)=2^{\frac{3\left(n^{2}-n+8\right)}{2}} \cdot 3^{\frac{3(n+8)(n-1)}{2}}$,

(vii) $H \Pi_{2}(G)=2^{3\left(n^{2}-n+8\right)} \cdot 3^{3(n+8)(n-1)}$,

(viii) $P C \Pi(G)=2^{\frac{-3\left(n^{2}-n+8\right)}{4}} \cdot 3^{\frac{-3(n+8)(n-1)}{4}}$.

\subsection{Multiplicative topological indices of the circumcoronene se- ries of benzenoid $\left(H_{k}, k \geq 1\right)$.}

Finally, we aim to determine the multiplicative degree based indices for circumcoronene series of benzenoid. Its vertex and edge partitions are shown in Table 4.7 and Table 4.8, respectively.

Table 4.7: Vertex partition of the circumcoronene series of benzenoid $H_{k}$.

\begin{tabular}{ccc}
\hline Partition of $V(G)$ & $V_{2}$ & $V_{3}$ \\
\hline Frequency & $6 k$ & $6 k(k-1)$ \\
\hline
\end{tabular}

Table 4.8: Edge partition of the circumcoronene series of benzenoid $H_{k}$.

\begin{tabular}{cccc}
\hline Partition of $E(G)$ & $E_{4} \cap E_{4}^{*}$ & $E_{5} \cap E_{6}^{*}$ & $E_{6} \cap E_{9}^{*}$ \\
\hline Frequency & 6 & $12(k-1)$ & $9 k^{2}-15 k+6$ \\
\hline
\end{tabular}

Thus, using the vertex and edge partitions for the circumcoronene series of benzenoid $\left(H_{k}\right)$ in the formulation of multiplicative topological indices, we obtain the following theorem as before. 
Theorem 4. Let $G$ be the circumcoronene series of benzenoid $\left(H_{k}\right)$. Then we have

(i) $W_{1}^{s}(G)=2^{(6 k s)} \cdot 3^{6 k s(k-1)}$,

(ii) $M Z_{1}^{a}(G)=2^{3 a\left(3 k^{2}-5 k+6\right)} \cdot 3^{3 a\left(3 k^{2}-5 k+2\right)} \cdot 5^{12 a(k-1)}$,

(iii) $M Z_{2}^{a}(G)=2^{(12 a k)} \cdot 3^{18 a k(k-1)}$,

(iv) $A B C(G)=2^{3\left(3 k^{2}-7 k+3\right)} \cdot 3^{-3\left(3 k^{2}-5 k+2\right)}$,

(v) $G A H(G)=\left(\frac{2 \sqrt{6}}{5}\right)^{12(k-1)}$.

Now putting $s=1,2, a=1,2,-\frac{1}{2}$, in Theorem 4, we obtain the following corollary.

Corollary 4. Different multiplicative degree based indices for the circumcoronene series of benzenoid $\left(H_{k}\right)$ are given by,

(i) $N K(G)=2^{(6 k)} \cdot 3^{6 k(k-1)}$,

(ii) $\Pi_{1}(G)=2^{(12 k)} \cdot 3^{12 k(k-1)}$,

(iii) $\Pi_{1}^{*}(G)=2^{3\left(3 k^{2}-5 k+6\right)} \cdot 3^{3\left(3 k^{2}-5 k+2\right)} \cdot 5^{12(k-1)}$,

(iv) $H \Pi_{1}(G)=2^{6\left(3 k^{2}-5 k+6\right)} \cdot 3^{6\left(3 k^{2}-5 k+2\right)} \cdot 5^{24(k-1)}$,

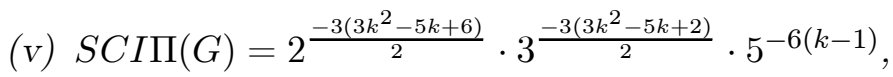

(vi) $\Pi_{2}(G)=2^{(12 k)} \cdot 3^{18 k(k-1)}$,

(vii) $H \Pi_{2}(G)=2^{(24 k)} \cdot 3^{36 k(k-1)}$,

(viii) $P C \Pi(G)=2^{(-6 k)} \cdot 3^{-9 k(k-1)}$.

\section{Conclusion}

Because of the controversial evolution of viruses, many new diseases can be discovered every year. That is why, new drug design is required to treat new diseases. Topological index play vital role to design novel drug as per diseases. In this work, we have put some efforts to obtain some multiplicative degree based topological indices of certain molecular graphs that widely appear in drug structures. Further, the results are plotted 

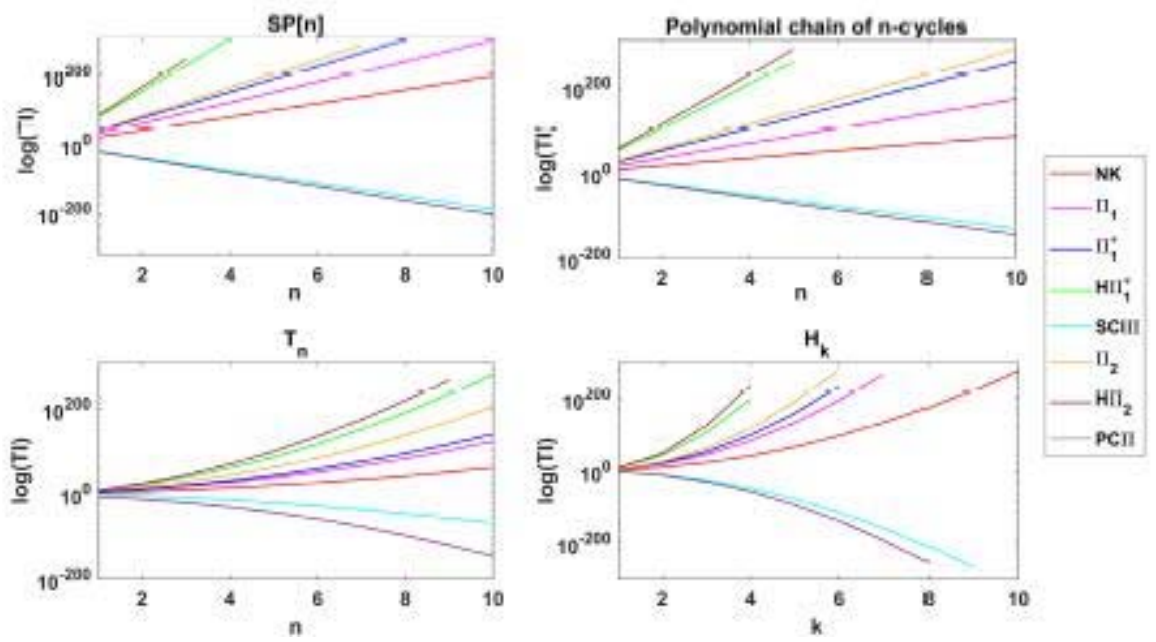

Figure 4.1: Plotting of topological indices against their parameters. By $\log (\mathrm{TI})$, we mean the logarithm of topological indices.

against their parameters in Figure 4.1. These theoretical results might be helpful to understand the underlying topology of the aforesaid chemical structures. As a future work, we would like to continue this work for some nanostar dendrimers. 


\section{References}

[1] J. Devillers and A. T. Balaban, Eds., Topological indices and related descriptors in QSAR and QSPR. Amsterdam: Gordon \& Breach, 1999.

[2] W. Gao, W. Wang, and M. R. Farahani, "Topological indices study of molecular structure in anticancer drugs", Journal of chemistry, vol. 2016, Art ID. 321632, 2016, doi: 10.1155/2016/3216327

[3] Y. C. Kwun, A.R. Virk, W. Nazeer, M. A. Rehman, and S. M. Kang, "On the multiplicative degree-based topological indices of silicon-carbon $\mathrm{Si}_{2} \mathrm{C}_{3}-\mathrm{I}[\mathrm{p}, \mathrm{q}]$ and $\mathrm{Si}_{2} \mathrm{C}_{3}-\mathrm{II}$ [p,q]", Symmetry, vol. 10, no. 8, Art ID. 320, Aug. 2018, doi: 10.3390/sym 10080320

[4] G. E. Mehak and A. H. Bhatti, "Zeroth- order general Randić index of line graphs of some chemical structures in drugs", Scientific Bulletin "Politehnica" University of Bucharest. Series B, Chemistry and materials science (Online), vol. 81, no. 1, pp. 47-70, 2019. [On line]. Available: https:// bit.ly/3cuHLxq

[5] W. Gao, M. K. Siddiqui, M. Imran, M. K. Jamil, and M. R. Farahani, "Forgotten topological index of chemical structure in drugs", Saudi pharmaceutical journal, vol. 24, no. 3, pp. 258-264, May. 2016, doi:10.1016/ j.jsps.2016.04.012

[6] I. Gutman, "Multiplicative Zagreb indices of trees", Bulletin of International Mathematical Virtual Institute, vol. 1, pp. 13-19, 2011. [On line]. Available: https:// bit.ly/3j7hkjZ

[7] M. Ghorbani and N. Azimi, "Note on multiple Zagreb indices", Iranian journal of mathematical chemistry, vol. 3, no. 2, pp. 137-143, 2012, doi: 10.22052/ IJMC.2012.5233

[8] W. Gao, M. Kamran Jamil, W. Nazeer, and M. Amin, "Degree-Based Multiplicative Atom-bond Connectivity Index of Nanostructures", IAENG International journal of applied mathematics, vol. 47, no.4, pp. 388-397, 2017. [On line]. Available: https:// bit.ly/2Ez6yEd

[9] S. Mondal, M. A. Ali , N. De, and A. Pal, "Bounds for neighborhood Zagreb index and its explicit expressions under some graph operations", Proyecciones (Antofagasta, On line), vol. 39, no. 4, pp. 799-819, Aug. 2020, doi: 10.22199/ issn.0717-6279-2020-04-0050 
[10] M. Cancan, I. Ahmad, and S. Ahmad, "Molecular descriptors of certain OTIS interconnection networks", Proyecciones (Antofagasta, On line), vol. 39, no. 4, pp. 769-786, Aug. 2020, doi: $10.22199 /$ issn.0717-6279-2020-04-0048

[11] A. J. M. Khalaf, A. Javed, M. K. Jamil, M. Alaeiyan, and M. Reza Farahani, "Topological properties of four types of porphyrin dendrimers", Proyecciones (Antofagasta, On line), vol. 39, no. 4, pp. 979-993, Aug. 2020, doi: 10.22199/ issn.0717-6279-2020-04-0061

[12] S. Mondal, N. De, and A. Pal, "Multiplicative degree based topological indices of nanostar dendrimers", Biointerface research in applied chemistry, vol. 11, no. 1, pp. 7700-7711, 2021, doi: 10.33263/ BRIAC1 11.77007711

[13] S. Wang and B. Wei, "Multiplicative Zagreb indices of k-trees", Discrete applied mathematics, vol. 180, pp. 168-175, Jan. 2015, doi: 10.1016/ j.dam.2014.08.017

[14] H. Narumi and M. Katayama, "Simple topological index: a newly devised index characterizing the topological nature of structural isomers of saturated hydrocarbons", Memoirs of the Faculty of Engineering, Hokkaido University, vol. 16, no. 3, pp. 209-214, 1984. [On line]. Available: https:/ / bit.ly/366Nzw7

[15] I. Gutman, B. Rusci, N. Trinajsti, and C. F. Wilcox, Jr. "Graph theory and molecular orbitals. XII. Acyclic polyenes", Journal of chemical physics, vol. 62, no. 9, pp. 3399-3405, 1975, doi: 10.1063/1.430994

[16] V. R. Kulli, B. Stone, S.Wang, and B. Wei, "Generalised multiplicative indices of polycyclic aromatic hydrocarbons and benzenoid systems", Zeitschrift fur naturforschung A, vol. 72, no. 6, pp. 573-576, 2017, doi: 10.1515/ zna-2017-0104

[17] M. Eliasi, A. Iranmanesh, and I. Gutman, "Multiplicative versions of first Zagreb index", MATCH Communications in mathematical and in computer chemistry, vol. 68, no. 1, pp. 217-230, 2012. [On line]. Available: https:/ / bit.ly/33UtOVO

[18] V. R. Kulli, "Multiplicative Hyper-Zagreb indices and coindices of graphs: computing these indices of some nanostructures", International research journal of pure algebra, vol. 6, no. 7, 2016. [On line]. Available: https:// bit.ly/2GbmQTY

[19] V. R. Kulli, "Multiplicative connectivity indices of TUC4 $\mathrm{C}_{8}[\mathrm{~m}, \mathrm{n}]$ and TUC4 [m, n] nanotubes", Journal of computer and mathematical sciences, vol. 7, no. 11, pp. 599-605, 2016. [On line]. Available: https:// bit.ly/3cuXBZb 
[20] N. Nishiyama and K. Kataoka, "Polymeric micelle drug carrier systems: PEG-PAsp(Dox) and second generation of micellar drugs," in Polymer drugs in the clinical stage, H. Maeda, A. Kabanov, K. Kataoka and T. Okano, Eds. Boston, MA: Springer, 2004, pp. 155-177, doi: 10.1007/0-306-47932-X_10

[21] S. A. Senevirathne, K. E. Washington, M. C. Biewer, and M. C. Stefan, "PEG based anti-cancer drug conjugated prodrug micelles for the delivery of anti-cancer agents", Journal of materials chemistry $B$, vol. 4, no. 3, pp. 360-370, 2016, doi: 10.1039/C5TB02053K

[22] Y. Masayuki, M. Mizue, Y. Noriko, O. Teruo, S. Yasuhisa, K. Kazunori, and I. Shohei, "Polymer micelles as novel drug carrier: Adriamycincon jugated poly(ethylene glycol)-poly(aspartic acid) block copolymer", Journal of controlled release, vol. 11, no. 2-3, pp. 269-278, Jan. 1990, doi: 10.1016/ 0168-3659(90)90139-K

[23] J. Rada, "The linear chain as an extremal value of VDB topological indices of polyomino chains", Applied mathematical sciences, vol. 8, no. 103,pp. 5133-5143, 2014, doi: 10.12988/ams.2014.46507

[24] P. W. Kasteleyn, "The statistics of dimers on a lattice: I. The number of dimer arrangements on a quadratic lattice", vol. 27, no. 12, Physica, pp. 1209-1225, Dec. 1961, doi: 10.1016/0031-8914(61)90063-5

[25] J. R. Dias, "Correlations and topology of triangular benzenoid hydrocarbons: a comparative study of two series representing the least and most stable benzenoid hydrocarbons", Journal of physical organic chemistry, vol. 15, no. 2, pp.94-102, 2002, doi: 10.1002/ poc.455 